\title{
Totally Greedy Coin Sets and Greedy Obstructions
}

\author{
L.J. Cowen Robert Cowen* Arthur Steinberg \\ Department of Mathematics \\ Queens College, CUNY
}

\author{
Submitted: May 24, 2008; Accepted: Jul 5, 2008; Published: Jul 14, 2008 \\ Mathematics Subject Classifications: 05A99, 68R05, 90C27
}

\begin{abstract}
A coin set is a strictly increasing list of positive integers that always begins with 1. A coin set is called greedy when the simple greedy change-making algorithm always produces the fewest number of coins in change. Here, the greedy changemaking algorithm repeatedly selects the largest denomination coin less than the remaining amount until it has assembled the correct change. Pearson has provided an efficient algorithm for determining whether a coin set is greedy. We study a stricter property on coin sets, called total greediness, which requires that all initial subsequences of the coin set also be greedy, and a simple property makes it easy to test if a coin set is totally greedy. We begin to explore the theory of greedy obstructions- those coin sets that cannot be extended to greedy coin sets by the addition of coins in larger denominations.
\end{abstract}

\section{Introduction}

One well-known property of US coin denominations comes from the famous "change making problem." In particular, suppose a cashier needs to return a value of $c$ cents change to a customer. The cashier has pennies (worth 1 cent), nickels (worth 5 cents), dimes (worth ten cents) and quarters (worth 25 cents). There may be several different ways to represent $c$ cents using this coin set; for example, eleven cents could be made using eleven pennies, or two nickels and a penny, or a dime and a penny. We call a representation of $c$ cents optimal if it uses the fewest number of coins possible; so the dime and penny is the optimal representation of eleven cents in the US coin set. In general, for a coin set $C$ with denominations $\left(a_{1}, a_{2}, a_{3}, \ldots a_{k}\right)$ we denote by $N[C, y]$ the number of coins in an optimal representation of $y$ using coin set $C$, or just $N[y]$ when $C$ is clear from context. In what follows, we restrict ourself to coin sets $C$ where $a_{1}=1$; so that $N[C, y] \leq y$ is finite for

*Corresponding author: robert.cowen@gmail.com 
all values $y$. Elements of $C$ will, naturally, be referred to as "coins," and in what follows, we will always list the coins in increasing order so that $1<a_{2}<a_{3} \ldots<a_{k}$.

The following simple greedy algorithm will produce an optimal representation of any value $c$ for the US coin set $(1,5,10,25)$. Choose as many quarters as possible without exceeding the value $c$, then choose as many dimes as possible to make up the remainder, then choose as many nickels as possible, and finally make up the rest with pennies. However, it is easy to construct coin sets for which this greedy algorithm fails to always produce an optimal representation; for example if the coin set is $(1,4,5)$, the greedy algorithm uses four coins to construct 8 cents $(5+1+1+1)$, but $N[8]=2(4+4)$. The number of coins used to construct $c$ cents greedily in coin set $C$ will be denoted by $G[C, c]$ or $G[c]$ when $\mathrm{C}$ is fixed.

We will use the following notation. If $C=\left(1, a_{2}, \ldots, a_{k}\right)$ is a coin set, then $r_{1} \mathbf{1} \oplus$ $r_{2} \mathbf{a}_{\mathbf{2}} \oplus \ldots \oplus r_{k} \mathbf{a}_{\mathbf{k}}$ will be used to denote the multiset consisting of $r_{1}$ coins of denomination $1, r_{2}$ coins of denomination $a_{2}$, up to $r_{k}$ coins of denomination $a_{k}$.

Definition 1.1 If $C=\left(1, a_{2}, a_{3}, \ldots, a_{k}\right)$ is a coin set and $x$ is a positive integer, we define the greedy representation for $x$ recursively as follows. Let $i$ be the largest index such that $a_{i} \leq x$. Then the greedy representation of $x$ is $\mathbf{a}_{\mathbf{i}} \oplus\{$ the greedy representation of $x-a_{i}$ \}. The number of coins used to construct $x$ cents greedily in coin set $C$ will be denoted by $G[C, x]$ or $G[x]$ when $C$ is fixed.

Definition $1.2 A$ coin set $C=\left(1, a_{2}, a_{3}, \ldots, a_{k}\right)$ is greedy if the greedy algorithm produces an optimal representation of $c$ for all positive integer values, $c$.

Greedy coin sets are termed "canonical" in [2] and [4]. If the optimal representation of $c$ uses fewer coins than the greedy representation, $c$ will be referred to as a counterexample to greediness. The following theorem is due to Kozen and Zaks [2].

Theorem 1.3 If coin set $\left(1, a_{2}, a_{3}, \ldots, a_{k}\right)$ is not greedy, there is a counterexample $c$, with $a_{3}+1<c<a_{k}+a_{k-1}$.

Obviously the search for a counterexample could be quite long, if the coins are large. Pearson [4] has provided a polynomial algorithm for determining whether a coin set is greedy, that runs in $O\left(k^{3} \log ^{2} a_{k}\right)$ time, where $k$ is the number of coins in the coin set and $a_{k}$ is the largest coin; if $a_{k}$ is bounded by a constant, it runs in $O\left(k^{3}\right)$ time. In this paper, we investigate a stricter property of coin sets, called total greediness which is defined as follows:

Definition $1.4 A$ coin set $\left(1, a_{2}, a_{3}, \ldots, a_{k}\right)$ is totally greedy if for each $i, 1 \leq i \leq k$, the coin set $\left(1, \ldots, a_{i}\right)$ is greedy.

That is, not only is the coin set greedy, but each initial segment of the coin set is also (totally) greedy.

Lemma 1.5 There are greedy coin sets that are not totally greedy. 
Proof. The proof is by construction. Let $C=(1,2,4,5,8)$. To show $C$ is greedy, one only has to check that there is no counterexample, $c$, where $5<c<13$, by Theorem 1.3. This is a small number of values and is easily checked by hand. Alternatively, one can use Stan Wagon's implementation of Pearson's algorithm in Mathematica $^{T M}$ [6] or his elegant implementation for small examples [7]. However, $(1,2,4,5)$ is not greedy because the optimal representation of 8 is $2 \cdot \mathbf{4}$ ( 2 coins), while the greedy representation is $\mathbf{5} \oplus \mathbf{2} \oplus \mathbf{1}$ (3 coins).

In this paper, we present a $O\left(k^{2} \log ^{2} a_{k}\right)$-time algorithm to determine if a coin set $C=\left(1, a_{2}, a_{3}, \ldots, a_{k}\right)$ is totally greedy. We are also inspired by Lemma 1.5 to investigate further when adding additional coins in larger denominations can restore the greedy property to a non-greedy coin set, and when they cannot. This motivates the following definition:

Definition $1.6 A$ coin set $\left(1, a_{2} \ldots a_{k}\right)$ is a greedy obstruction or simply an obstruction if and only if the coin set is not greedy, and no coin set which extends $\left(1, a_{2}, \ldots a_{k}\right)$ by appending a finite set of coins of denominations larger than $a_{k}$ can be greedy. A minimal obstruction is an obstruction that has no proper subsequence that is an obstruction.

\section{The Greedy Extension Theorem}

In this section, we prove a theorem about totally greedy coin sets and exhibit an algorithm that tests whether a coin set is totally greedy. The theorem turns out to be equivalent to a result of Magazine, Nemhauser and Trotter [3], but an independent proof is supplied for completeness.

Lemma 2.1 $N[x+y] \leq N[x]+N[y]$.

Proof. Follows from the fact that an optimal representation for $\mathrm{x}$ when added to a optimal representation for $\mathrm{y}$ is a representation for $\mathrm{x}+\mathrm{y}$.

If $x$ is a rational number, $\lceil x\rceil$ denotes the ceiling of $x$, that is, the smallest integer greater than or equal to $x$.

Theorem 2.2 The Greedy Extension Theorem [GET]. Suppose $C_{1}=\left(a_{1}, a_{2} \ldots a_{k}\right)$ is greedy and $C_{2}=\left(a_{1}, a_{2} \ldots a_{k}, a_{k+1}\right)$. Let $m=\left\lceil a_{k+1} / a_{k}\right\rceil$. Then $C_{2}$ is greedy if and only if $G\left[C_{2}, m a_{k}\right] \leq m$.

Proof. Let $G_{i}[y]$ denote the number of coins in the greedy representation of $y$ using coin set $C_{i}, i=1,2$. Clearly, $C_{2}$ is greedy only if $G_{2}\left[m a_{k}\right] \leq m$.

Conversely, suppose that $G_{2}\left[m a_{k}\right] \leq m$. We note that $G_{1}$ is "subadditive"; that is, $G_{1}[x+y] \leq G_{1}[x]+G_{1}[y]$, by the lemma above, since $C_{1}$ is greedy. We relabel $a_{k}=a$, $a_{k+1}=b$. Then $G_{2}[m a] \leq m$ is true if and only if $G_{1}[m a-b]<m$; this is so because 
$m a=b+(m a-b)$, so $G_{2}[m a]=1+G_{2}[m a-b]=1+G_{1}[m a-b]$ where this last equality follows since $m a-b<a$, by the minimality of $m$.

We must show that $C_{2}$ is greedy, that is, for any positive integer $y, G_{2}[y]=N\left[C_{2}, y\right]$. Let $[y]_{i}$ denote the greedy representation of $y$ using coin set $C_{i}, i=1,2$. By way of contradiction, let $x$ be the minimal counterexample for $C_{2}$, that is, the smallest positive integer for which the greedy algorithm does not produce the optimal representation. Clearly, we can assume that $x>b$. Then, for some nonnegative integers $k, l$ and $s$, we can represent $[x]_{2}$ as $k \mathbf{b} \oplus l \mathbf{a} \oplus[s]_{2}=k \mathbf{b} \oplus l \mathbf{a} \oplus[s]_{1}$ since $s<a$; so $G_{2}[x]=k+l+G_{1}[s]$.

If there was another way to represent $x$ using fewer than $G_{2}[x]$ coins, then this representation cannot also begin with $k \mathbf{b}$; for, otherwise the reduction must come from $l \mathbf{a} \oplus[s]_{1}$ which then would also be representable using fewer than $G_{1}[x-k b]$ coins in $C_{1}$. However this would contradict that $C_{1}$ is a greedy coin set.

Hence the representation must remove $q$ coins of denomination $\mathbf{b}$, for some $q>0$ and then to compensate for these $q$ coins, an optimal representation of $q b$ using only the coins from $C_{1}$ must be found. Next, $b=(m-1) a+r, 0<r \leq a$. Since $C_{1}$ is greedy, the optimal representation for $x$ must begin with $(k-q)$ coins of denomination $\mathbf{b}$, followed by $l+q(m-1)$ coins of denomination $\mathbf{a}$, followed by $[q r+s]_{1}$, the greedy representation of $q r+s$ in coin set $C_{1}$.

Let $D$ be the difference between the number of coins in the optimal representation of $x$ and the greedy representation of $x$ using coin set $C_{2}$. Then $x$ being a counterexample to the theorem implies that $D<0$. We have

$$
D=-q+q(m-1)+G_{1}[q r+s]-G_{1}[s] .
$$

Since $b=(m-1) a+r, m a-b=a-r$. Since $m a=b+(m a-b)$ and $m a-b<a$ and $G_{2}[m a] \leq m$, it follows that $m \geq G_{2}[m a]>G_{2}[m a-b]=G_{1}[m a-b]$, where the last equality follows from $m a-b<a$.

Thus for some positive integer $t$, we can write $m=G_{1}[m a-b]+t=G_{1}[a-r]+t$. Substituting for $m$ in Equation 1, we have $D=-q+q\left(G_{1}[a-r]+t-1\right)+G_{1}[q r+s]-G_{1}[s]$. Since $s<a$, we can rewrite $q+G_{1}[s]$ as $G_{1}[s+q a]$. Also, $q G_{1}[a-r] \geq G_{1}[q(a-r)]$ by subadditivity. Therefore $D \geq q\left(t-1+G_{1}[q(a-r)]+G_{1}[q r+s]-G_{1}[s+q a]\right.$. However, $q(a-r)+(q r+s)=s+q a$, and so again, by subadditivity, $G_{1}[s+q a] \leq G_{1}[q(a-r)]+$ $G_{1}[q r+s]$; thus $G_{1}[q(a-r)]+G_{1}[q r+s]-G_{1}[s+q a] \geq 0$. This together with $t \geq 1$ implies that $D \geq 0$, a contradiction. Thus the greedy representation uses the minimal number of coins and is optimal.

We remark that Theorem 2.2 is equivalent to a special case of a theorem proved by Magazine, Nemhauser and Trotter in 1975 [3]. The equivalence follows by setting all the $c_{i}=1$ in their Theorem 1 , and noting that $G_{2}\left[m a_{k}\right]=1+G_{1}\left[m a_{k}-a_{k+1}\right]$.

Corollary 2.3 Coin set $(1,2, b)$ is greedy for any $b>2$.

Proof. (1) and $(1,2)$ are surely greedy. Let $m=\lceil b / 2\rceil$. Clearly, $m \geq 2$. If $b$ is even, $2 m=b$ and $G[2 m]=1<m$. If $b$ is odd, $2 m=b+1$; hence, in this case, $G[2 m]=2 \leq m$. 
Corollary $2.4(1,3,5 \ldots, 2 k-1)$ is greedy, for each positive integer $k$.

Proof. The smallest integer $m$ such that $m(2 k-3) \geq(2 k-1)$ is $m=2$, but, $2(2 k-3)=$ $(2 k-1)+(2 k-5)$.

Corollary $2.5(1, a, 2 a-1)$ is greedy, for any $a>1$.

Proof Let $m=\lceil(2 a-1) / a\rceil$, so $m=2$. But then, $m a=(2 a-1)+1$ and the coin set is greedy by GET.

Corollary 2.6 Let $m=\lceil b / a\rceil$. Then $(1, a, b)$ is (totally) greedy if and only if $m a-b<m$.

Proof. $(1, a)$ is totally greedy. $m a=b+(m a-b)$ and $(m a-b)<a$, since $(m-1) a<b$. Thus $G[m a]=1+(m a-b) \leq m$ if and only if $(1, a, b)$ is a totally greedy coin set.

Corollary 2.7 Suppose $m=\lceil b / a\rceil$ and $b=(m-1) a+r$, for some $0<r \leq a$. Then, $(1, a, b)$ is greedy iff $a-r \leq m-1$.

Proof. By Corollary 2.6, with $b=(m-1) a+r,(1, a, b)$ is greedy iff $1+m a-((m-1) a+r) \leq$ $m$. This is equivalent to $1+a-r \leq m$, that is, $a-r \leq m-1$.

Corollary 2.8 (Kozen and Zaks [2]) Coin set $(1, a, b)$ with $b=a q+r, 0 \leq r<a$ is (totally) greedy if and only if $r=0$ or $a-q \leq r$.

Proof. If $r=0, q a=b$ and $(1, a, b)$ is greedy. Otherwise, since $b=a q+r$, and $0<r<a$, $q=m-1$ and the result follows from Corollary 2.7.

Corollary 2.9 Suppose $m=\lceil b / a\rceil$ and suppose that $b=(m-1) a+r, \quad 0<r \leq a$. Then, $(1, a, b)$ is greedy iff $b>(a-r)(a-1)$.

Proof. Suppose first that $(1, a, b)$ is greedy. Then, by Corollary 2.6, $m>(m a-b)$ or $b>m(a-1)$. Also, by Corollary 2.7, $m>(a-r)$. Hence, by transitivity, $b>(a-r)(a-1)$.

Conversely, suppose that $b>(a-r)(a-1)$. If $m>(a-r)$, then $(1, a, b)$ is greedy by Corollary 2.7. Suppose then that $m \leq(a-r)$. Equivalently, $m \leq m a-b$, or $b \leq m(a-1)$. However, in this case, $b \leq(a-r)(a-1)$, a contradiction.

Corollary $2.10\left(1, a,(a-1)^{2}\right)$ is not greedy.

Proof. We make use of Corollary 2.9. $b=(a-1)^{2}$ implies $m=a-1$ and hence $r=1$. Thus $b=(a-r)(a-1)$.

Corollary 2.11 If $b>(a-1)^{2}$, then $(1, a, b)$ is greedy. 
Proof.We make use of Corollary 2.9. Since $r>0, b>(a-1)^{2}$ implies $b>(a-r)(a-1)$.

The preceding two corollaries imply that $(1,3, b)$ is greedy iff $b \neq 4$. Also, it is easily seen, using Corollary 2.9 , that $(1,4,5),(1,4,6),(1,4,9)$ aren't greedy, while Corollary 2.11 implies that $(1,4, b)$ is greedy if $b>9$; thus $(1,4, b)$ is greedy iff $b \neq 5,6,9$. For $(1,5, b)$ the situation is somewhat more complicated. If $b>16$, the triple is greedy by Corollary 2.11. Moreover it is easy to show, using Corollary 2.9 , that $(1,5,6),(1,5,7),(1,5,8),(1,5,11)$, and $(1,5,12)$ are not greedy, while $(1,5,9)$ and $(1,5,10),(1,5,13),(1,5,14)$ and $(1,5,15)$ are greedy. Hence $(1,5, b)$ is greedy iff $b \neq 6,7,8,11,12$.

The Greedy Extension Theorem also leads to an easy method to construct a variety of arbitrarily large (totally) greedy coin sets.

Corollary 2.12 Suppose $\left(a_{1}, a_{2}, a_{3}\right)$ is (totally) greedy and for $i \geq 3, a_{i+1}=m a_{i}-$ $\left(w_{1} a_{i-1}+w_{2} a_{i-2}\right)$, where $m$ is an integer greater than 1, and the $w$ 's are non-negative integers satisfying

(1) $w_{1}+w_{2} \leq m-1$

(2) $w_{1} a_{i-1}+w_{2} a_{i-2}<a_{i}$,

(3) $w_{2} a_{i-2}<a_{i-1}$,

Then any finite initial segment of $\left(a_{i}\right)_{i=1}^{\infty}$ is a totally greedy coin set.

Proof. We make use of induction and GET. By hypothesis, $\left(a_{1}, a_{2}, a_{3}\right)$ is totally greedy. Assume $C_{1}=\left(a_{1}, a_{2} \ldots a_{i}\right)$ is totally greedy, $i \geq 3$, and $C_{2}=\left(a_{1}, a_{2} \ldots a_{i}, a_{i+1}\right)$; then $m a_{i}=a_{i+1}+\left(w_{1} a_{i-1}+w_{2} a_{i-2}\right)$. Clearly, $m a_{i} \geq a_{i+1}$; also, $(m-1) a_{i}=m a_{i}-a_{i}=$ $a_{i+1}+\left(\left(w_{1} a_{i-1}+w_{2} a_{i-2}\right)-a_{i}\right)<a_{i+1}$, by (2). Hence $m=\left\lceil a_{i+1} / a_{i}\right\rceil$. In addition, $G\left[C_{2}, m a_{i}\right]=1+G\left[C_{2},\left(w_{1} a_{i-1}+w_{2} a_{i-2}\right)\right]$, by condition $(2)$; however, $G\left[C_{2},\left(w_{1} a_{i-1}+\right.\right.$ $\left.\left.w_{2} a_{i-2}\right)\right]=w_{1}+w_{2}$, by conditions (2) and (3). Hence, $G\left[C_{2}, m a_{i}\right]=1+w_{1}+w_{2} \leq m$, by condition (1). Therefore $C_{2}$ is totally greedy, if $C_{1}$ is totally greedy, by GET. It follows, by induction, that $\left(a_{i}\right)_{i=1}^{k}$ is totally greedy for all $k \geq 3$; also, since $\left(a_{1}, a_{2}, a_{3}\right)$ is totally greedy, any finite initial segment of $\left(a_{i}\right)_{i=1}^{\infty}$ is a totally greedy coin set.

If any finite initial segment of $\left(a_{i}\right)_{i=1}^{\infty}$ is a totally greedy coin set, we will call $\left(a_{i}\right)_{i=1}^{\infty}$ a tg-sequence. Corollary 2.12 immediately shows that the Fibonacci sequence is a tgsequence; $a_{i+1}=2 a_{i}-a_{i-2}$ with $\left(a_{0}=1\right), a_{1}=1, a_{2}=2, a_{3}=3$ satisfies the conditions of Corollary 2.12 and is an equivalent definition of the Fibonacci sequence. Other appropriate linear recurrences give other (less familiar) tg-sequences, for example setting $a_{i+1}=7 a_{i}-3 a_{i-1}-2 a_{i-2}$, with $a_{1}=1, a_{2}=3$ and $a_{3}=5$ yields the tg-sequence: $(1,3,5,24,147,947,6140, \ldots)$.

\section{Testing for Total Greediness}

The greedy extension theorem provides the basis for an algorithm for deciding whether a coin set is totally greedy, which as we shall show, runs in $O\left(k^{2} \log ^{2} a_{k}\right)$, where $k$ is the 
number of coins in the coin set and $a_{k}$ is the value of the largest coin. The algorithm is based on the following theorem:

Theorem 3.1 Let $C=\left(1, a_{2}, \ldots, a_{k}\right)$ be a coin set with $k$ coins. Suppose that $C_{i}=$ $\left(1, a_{2}, \ldots, a_{i}\right)$ and $m_{i}=\left\lceil a_{i+1} / a_{i}\right\rceil, 2 \leq i<k$. Then $C$ is totally greedy if and only if $G\left[C_{i+1}, m_{i} a_{i}\right] \leq m_{i}$, for $2 \leq i<k$.

Proof. If $C$ is totally greedy, then each $C_{i+1}$ is greedy and so $G\left[C_{i+1}, m_{i} a_{i}\right] \leq m_{i}, 2 \leq$ $i<k$. So suppose that $G\left[C_{i+1}, m_{i} a_{i}\right] \leq m_{i}, 2 \leq i<k$. Clearly, $\left(1, a_{2}\right)$ is greedy and now GET together with $G\left[C_{i+1}, m_{i} a_{i}\right] \leq m_{i}$ implies that if $C_{i}$ is greedy then $C_{i+1}$ is greedy. It follows, by induction, that $C_{i}$ is greedy, $2 \leq i \leq k$, that is, $C$ is totally greedy.

Theorem 3.2 Let $C=\left(1, a_{2}, \ldots, a_{k}\right)$ be a coin set with $k$ coins and let $a_{k}$ be the size of the largest coin. Then it can be determined if $C$ is totally greedy in $O\left(k^{2} \log ^{2} a_{k}\right)$ time.

Proof. Let $C_{i}$ be the coin set consisting of the first $i$ coins of $C$. By Theorem 3.1, $C$ is totally greedy if and only if for each $i, 2 \leq i \leq k-1, G\left[C_{i+1}, m_{i} a_{i}\right] \leq m_{i}$, where $m_{i}=\left\lceil a_{i+1} / a_{i}\right\rceil$. When $i=k-1$, to test this condition, we first need to determine the value of $m_{k-1}$, which can be obtained by taking the ceiling of $a_{k} / a_{k-1}$. Note that $m_{k-1}$ is certainly less that $a_{k}$. Thus $m_{k-1} \cdot a_{k-1}$ is less than $a_{k}^{2}$ and thus has at most $\log a_{k}^{2}=2 \log a_{k}$ digits. To determine the greedy representation of $m_{k-1} \cdot a_{k-1}$, one simply applies the division algorithm and rewrites $m_{k-1} \cdot a_{k-1}$ as $x_{k} a_{k}+r_{k}$, and then sets $r_{k-1}=r_{k}$ and $x_{k-1}=0$ if $r_{k}<a_{k-1}$; otherwise $r_{k-1}$ is rewritten recursively as $x_{k-1} a_{k-1}+r_{k-2}$, and $r_{k-2}$ is expanded. Continuing down to $r_{1}, G\left[C_{k}, m_{k-1} a_{k-1}\right]=\sum_{i=1}^{k} x_{k}$. Thus to check the condition of the greedy extension theorem on $C_{k}$, when assuming $C_{i}$ also satisfies the condition for all $i<k$ requires at most $k+1$ integer division operations, each on integers of size at most $O\left(\log a_{k}\right)$. Here we use the simple upper bound on the complexity of integer division, bounding it by $O\left(q^{2}\right)$, for $q$-digit integers [1]. We check the condition first on $C_{1}$, then if it passes, on $C_{2}$, then if it passes, all the way up to $C_{k}$; each check costs less than the worstcase cost of checking the condition on $C_{k}$, so the cost of checking the condition for all $C_{i}$ is $\leq k$ times (the cost of checking the condition on $\left.C_{k}\right)=k O\left(k \log ^{2} a_{k}\right)=O\left(k^{2} \log ^{2} a_{k}\right)$.

Certainly the above algorithm for testing total greediness is better than the naive strategy of applying the fastest known algorithm for testing the ordinary greedy property (due to Pearson [4]) which runs in $O\left(k^{3} \log ^{2} a_{k}\right)$ time on each initial segment of the coin set, for a total running time of $O\left(k^{4} \log ^{2} a_{k}\right)$. (Pearson [4] proved his algorithm runs in $O\left(k^{3}\right)$ time, but he is assuming constant time integer multiplication and division; all results in this paper are stated in the bit model from whence we obtain the extra logarithmic factors.) Since our total greedy test also proves ordinary greediness, it can first be applied to a coin set whose (ordinary) greediness is of interest; if the coin set fails, the (more expensive) Pearson test for greediness can then be applied. 


\section{Short Greedy Sequences and Greedy Obstructions}

In this section, we begin to explore the relationship between greediness, total greediness, and begin to try to understand the theory of greedy obstructions for very short sequences. Although we have seen that not every greedy coin set is totally greedy, we can prove the following.

Theorem 4.1 Any greedy coin set with four elements is totally greedy.

Proof. Suppose false, that is, suppose that $(1, a, b, c)$ is greedy but $(1, a, b)$ is not greedy. Let $m=\lceil b / a\rceil$. Then $b=(m-1) a+r, 0<r \leq a-1$. Clearly $c \leq m a$, since $m a$ is a counterexample to the greediness of $(1, a, b)$ and it would persist as counterexample to $(1, a, b, c)$ if $c>m a$. Since, $c \leq m a, m$ is also the smallest integer such that $m a \geq c$; hence $c=(m-1) a+s, 0<s \leq a$. Clearly $r<s$, since $b<c$.

It follows that $c-b=s-r \leq a-1$. If $c-b=a-1$, then $2 b=c+(2 b-c)$. $2 b-c=b-(c-b)=b-(a-1)=(b-a)+1 \geq 2$ since $b-a \geq 1$, thus we know that the greedy representation of $2 b$ includes a coin of denomination $c$. We now show that $2 b-c$ cannot be represented by a single coin. Clearly $2 b-c \neq b$ and $2 b-c \neq c$. Also, $2 b-c \neq a$, since otherwise $b=2 a-1$ and $(1, a, b)$ would be greedy. Thus, $2 b$ is a counterexample to the greediness of $(1, a, b, c)$, since the greedy representation of $2 b$ now requires at least three coins rather than two.

If $c-b<a-1$, equivalently $b+a-c \geq 2$, then $b+a$ can be represented as $\mathbf{c} \oplus(b+a-c) \cdot \mathbf{1}$. Clearly, $b+a-c$ is not equal to $a, b$, or $c$. Hence, in this case, the greedy representation of $b+a$ in $(1, a, b, c)$ uses at least three coins rather than two.

Theorem 4.2 If $(1, a, b)$ is nongreedy, then so is $(1, a, b, c, d)$ for any integers $c, d$ with $b<c<d$.

Proof. Omitted for now, because this follows as a simple corollary of Theorem 4.5 which is proved below.

We want to better understand those coin sets that are greedy but not totally greedy. If the coins set has four elements we have seen that there aren't any. We look next at five element coin sets. We shall give a characterization of those five element coin sets that are greedy but not totally greedy, but first we need to prove the following lemma.

Lemma 4.3 If $U_{c}=(1, \ldots, a, b, c)$ is greedy, $U_{b}=(1, \ldots, a, b)$ is not greedy, but $U_{a}=$ $(1, \ldots, a)$ is greedy, then it must be the case that $b=a+r$ and $c=a+s$, where $0<r<$ $s \leq a$. Moreover, $2 b-c=a_{i}$, where $a_{i}$ is a coin, $1<a_{i}<a, a-a_{i}=s-2 r, a_{i}-r$ is $a$ coin, while $a-r$ is not a coin, and either $2 a=c$ or $2 a-c$ is a coin less than $a_{i}$.

Proof. Let $m=\lceil b / a\rceil$. Then $b=(m-1) a+r$, where $0<r \leq a-2$ (if $r=a-1$, $b=m a-1$ and $U_{b}$ would be greedy, by GET). If we adjoin $c$ to $U_{b}$ then we can assume that $c \leq m a$, since, otherwise, $m a$ is a counterexample to the greediness of $U_{b}$ by GET, and would persist as a counterexample for $U_{c}$. 
Clearly $m$ is also the smallest integer such that $m a \geq c$. Therefore, $c=(m-1) a+s$, where $r<s \leq a$. Thus, $2 b-c=(m-1) a+(2 r-s) \geq a+(2-a)=2$, since $m \geq 2$, $r \geq 1$, and $s \leq a$.

Now, $2 b=c+(2 b-c)$, so $G\left[U_{c}, 2 b\right]=1+G\left[U_{c}, 2 b-c\right]$. Therefore, $1+G[2 b-c] \leq 2$ since $U_{c}$ is greedy. It follows that $G\left[U_{c}, 2 b-c\right]=1$. Clearly $2 b-c \neq 1$. (Recall that $2 b-c \geq 2$.) Thus, $2 b-c$ is some coin, $a_{i}$, greater than 1 . Next we claim that $a_{i} \neq a$. Suppose this were false and $a_{i}=a$. First, $m=2$; otherwise, if $m>2, a_{i}=(m-1) a+(2 r-s)>$ $2 a+(2-a)>a$, which is impossible, assuming $a_{i}=a$. Since $m=2$ and still assuming that $a=a_{i}, a=a+(2 r-s)$, and so $2 r=s$; hence $b=a+r, c=a+2 r$. Now $U_{a}$ is greedy, but $U_{b}$ is not and $m=2$. Therefore, using GET, $2 a$ cannot be represented in $U_{b}$ with 2 coins. However $2 a=(a+r)+(a-r)=b+(a-r)$; thus $a-r$ is not a coin. $U_{c}$ is greedy and $c=a+2 r$. Thus, $a+b=2 a+r=(a+2 r)+(a-r)=c+(a-r)$, which implies that $a-r$ is a coin. Contradiction. Hence $a_{i} \neq a$. Also, since $2 b-c \geq 2, a_{i} \geq 2$. In addition, $s>2 r$ since $a-a_{i}=s-2 r$ and $a_{i}<a$. Hence $a-s=a_{i}-2 r$ and $a_{i} \geq 2 r$. Moreover $a_{i}-r$ is a coin since $a+s=2 a+2 r-a_{i}$ and $a+(a+r)=\left(2 a+2 r-a_{i}\right)+\left(a_{i}-r\right)=(a+s)+\left(a_{i}-r\right)$.

In a similar fashion we obtain conditions on $2 a-c$. For, $2 a=c+(2 a-c)$. Hence, $G\left[U_{c}, 2 a-c\right] \leq 1$ which implies either $2 a=c$ or $2 a-c$ is some coin $a_{j}$ with $a_{j}<a_{i}$ (since $a<b)$.

It is interesting to note that Lemma 4.3 also gives another proof of Theorem 4.1 , since if $(1, a, b, c)$ were greedy but $(1, a, b)$ were not greedy, then the lemma would imply the existence of coin $a_{i}$, between 1 and $a$.

Theorem 4.4 A coin set with five coins is greedy but not totally greedy iff it is of the form $(1,2, a, a+1,2 a)$, with $a>3$.

Proof. Suppose that $C=(1,2, a, a+1,2 a)$, with $a>3$. $(1,2, a)$ is greedy (Corollary 2.3); however $(1,2, a, a+1)$ is not greedy, if $a>3$, by the GET theorem, and the minimal counterexample is $2 a$. If $C$ was not greedy, the Kozen-Zaks Theorem gives bounds for a counterexample. The upper bound is $3 a$. Thus any counterexample $x$, to the greediness of $C$ must be of the form, $x=2 a+r, 0<r \leq a$. Clearly, $3 a=2 a+a$ cannot be a counterexample. Hence we can assume, $0<r<a$. If the optimal representation of $x$ uses $2 a$ it must be the same as the greedy representation since the remainder $r$ must be represented in the greedy coin set $(1,2, a)$, since $r<a$. Therefore the minimal counterexample must use two from $\{a, a+1\}$; however if it uses $a$ and $a+1$ say, $2 a+1$ could be replaced with $2 a$ and 1 and we are back to the previous case. Similarly for the other possibilities. Therefore $C$ must be greedy.

Suppose now that $U_{c}=\left(1, a_{2}, a, b, c\right)$ is greedy but not totally greedy. Then it must be that $U_{a}=\left(1, a_{2}, a\right)$ is greedy (by Theorem 4.2) and $U_{b}=\left(1, a_{2}, a, b\right)$ is not greedy. Hence by Lemma $4.3, b=a+r, c=a+s$, where $0, r<s \leq a$ and $s>2 r$. Moreover, $2 b-c=a_{i}, a_{i}$ a coin less than $a$ and greater than 1 . This means that $a_{i}=a_{2}$. In addition, $a-a_{i}=s-2 r$ and $a-r$ is not a coin, while $a_{i}-r$ is a coin and finally, either $2 a=c$ or $2 a-c$ is a coin less than $a_{i}$. 
By the above, $2 b-c=a_{2}$; now $a_{2}-r$, which is a coin, must be 1 . Hence $b=a+r=$ $a+a_{2}-1$ and $c=2 b-a_{2}=2 a+a_{2}-2$. So, $U_{c}=\left(a, a_{2}, a, a+a_{2}-1,2 a+a_{2}-2\right)$. Moreover, it cannot be that $2 a-c$ is a coin less than $a_{2}$, since this implies that $2 a-c=1$. But then, since $c=2 a+a_{2}-2$, it follows that $2-a_{2}=1$ and hence $a_{2}=1$, a contradiction. Hence $2 a=c$ and thus $a_{2}=2$.

Coin sets which are greedy, but not totally greedy, will be called conditionally greedy. If we consider those coin sets with five elements, the largest of which is 30, Theorem 4.4 shows that there are only 12 conditionally greedy coin sets in this range; we have calculated, using the Kozen-Zaks algorithm, that there 682 greedy coin sets (out of 23,751 coin sets) in this range. This means that there are 670 totally greedy coin sets of size five with largest coin, 30. Thus we feel it is relatively rare for a coin set to be conditionally greedy. We feel that this supports our strategy of testing for total greediness first when testing for greediness.

\subsection{Greedy Obstructions}

Recall that a greedy obstruction was defined to be a coin set that was not greedy, and furthermore could not be extended to a greedy coin set by the addition of a finite number of coins in larger denominations. Some sequences are trivially greedy obstructions. For instance, the sequence $(1,4,5,9)$ is a greedy obstruction because the optimal representation of 8 is $2 \cdot \mathbf{4}$ whereas the greedy representation is $\mathbf{5} \oplus 3 \cdot \mathbf{1}$ and clearly, no coins of denomination larger than $\mathbf{9}$ added to the coin set can be used to reduce the number of coins in the greedy representation of 8 . We call any such coin set which is not greedy, and there exists a greedy counterexample of size smaller than the largest denomination in the coin set a trivial greedy obstruction.

Nontrivial greedy obstructions are a more interesting class. (A non-trivial greedy obstruction is one where the smallest counterexample to greediness is larger than the largest coin in the coin set.) An example is the sequence $(1,3,4)$ - which certainly isn't greedy; moreover no finite sequence which begins $(1,3,4)$ can be greedy; this is a special case of the following theorem.

Theorem 4.5 Suppose $V=(1, a, b)$ is non-greedy. Then it is a (non-trivial, minimal) greedy obstruction.

Proof. We repeatedly use the following fact: $\left(^{*}\right)$ If $U$ is a non-greedy coin set with counterexample $x$ and $c$ is a coin adjoined to $U$ where $c>x$, then $U \cup\{c\}$ will be a trivial greedy obstruction.

Let $m=\lceil b / a\rceil$. Since $V$ is not greedy, $m \leq m a-b$, by Corollary 2.6.

We define four classes, $\mathcal{B}, \mathcal{C}, \mathcal{D}, \mathcal{E}$.

Let $\mathcal{B}$ be all coin sets $U=\left(1, a, b, \ldots, c_{k-1}, c_{k}\right)$ such that $c_{k}-c_{k-1}=b-a$. Note that $V \in \mathcal{B}$.

Let $\mathcal{C}$ be all coin sets $U=\left(1, a, b, \ldots, c_{k-1}, c_{k}\right)$ such that $c_{k}-c_{k-1} \leq a-1$.

Let $\mathcal{D}$ be all coin sets $U=\left(1, a, b, \ldots, c_{k-1}, c_{k}, c_{k+1}\right)$ such that $(\dagger) a \leq c_{k+1}-c_{k} \leq$ $b-a+1$ and $\left(1, a, b, \ldots, c_{k}\right)$ is non-greedy with counterexample $c_{k-1}+b$. 
Let $\mathcal{E}$ be the class of trivial obstructions.

We shall show that

(1) each of these classes consists of non-greedy coin sets (for $\mathcal{E}$ this is obvious).

(2) $\mathcal{B} \cup \mathcal{C} \cup \mathcal{D} \cup \mathcal{E}$ is closed under coin adjunction.

It follows immediately that $V$ is an obstruction.

First we show that coin sets in $\mathcal{B}$ are non-greedy. Let $U=\left(1, a, b, \ldots, c_{k-1}, c_{k}\right)$, where $c_{k}-c_{k-1}=b-a$. It then follows that $c_{k-1}+(m-1) a=c_{k}+(m a-b)$. However, $m a-b \geq m$ (Corollary 2.6) and $m a-b<a$ (by the minimality of $m$ ); hence $c_{k-1}+(m-1) a$ is representable by $m$ coins, whereas the above greedy representation requires at least $m+1$ coins.

Next suppose, $U=\left(1, a, b, \ldots, c_{k-1}, c_{k}\right)$ and $U \in \mathcal{C}$. There are two cases to consider: $c_{k}-c_{k-1} \leq a-2$ and $c_{k}-c_{k-1}=a-1$.

Case A. Assume that $c_{k}-c_{k-1} \leq a-2$. Here, we claim that $c_{k-1}+a$ is a counterexample to greediness. For, $c_{k-1}+a=c_{k}+\left(a+\left(c_{k-1}-c_{k}\right)\right)$. Now, $c_{k-1}+a$ is clearly representable by two coins, whereas $c_{k}+\left(a+\left(c_{k-1}-c_{k}\right)\right)$ is the greedy representation, since $a>a+\left(c_{k-1}-c_{k}\right)$ and needs at least three coins because $a+\left(c_{k-1}-c_{k}\right) \geq 2$, by our Case A assumption. Hence, any coin set in $\mathcal{C}$ satisfying Case $\mathrm{A}$ is non-greedy.

Case B. Assume $c_{k}-c_{k-1}=a-1$. Then, we claim that $c_{k-1}+b$ is a counterexample. For, $c_{k-1}+b=c_{k}+b+\left(c_{k-1}-c_{k}\right)=c_{k}+(b-a+1)$. Clearly, $b>b-a+1>1$, while $b-a+1$ is not a coin; for, if $b-a+1=a$, that is, if $b=2 a-1$, then $V$ would be greedy by Corollary 2.5. Thus the greedy representation of $c_{k-1}+b$ requires at least three coins rather than two. Hence any coin set in $\mathcal{C}$ is not greedy.

Next let $U=\left(1, a, b, \ldots, c_{k-1}, c_{k}, c_{k+1}\right), U \in \mathcal{D}$, that is $a \leq c_{k+1}-c_{k} \leq b-a+1$ and $c_{k-1}+b$ is a counterexample for $\left(1, a, b, \ldots, c_{k}\right)$. Since we have already shown that coin sets in $\mathcal{B}$ are not greedy, we can assume that $U \notin \mathcal{B}$, that is, $c_{k+1}-c_{k} \neq b-a$. We shall show that $c_{k}+b$ is a counterexample for $U$. For, $c_{k}+b=c_{k+1}+\left[\left(c_{k}+b\right)-c_{k+1}\right]$. Now by $(\dagger),\left[\left(c_{k}+b\right)-c_{k+1}\right] \geq a-1 \geq 2$; the last inequality follows since $a>3$, by Corollary 2.3, since $V$ is non-greedy. In addition, $\left[\left(c_{k}+b\right)-c_{k+1}\right]<b$, since $c_{k}-c_{k+1}<0$. Also $\left[\left(c_{k}+b\right)-c_{k+1}\right]$ is not a coin because $\left[\left(c_{k}+b\right)-c_{k+1}\right]=a$ is false since we are assuming that $U \notin \mathcal{B}$ and thus $c_{k+1}-c_{k} \neq b-a$. Hence the greedy representation requires at least three coins rather than two.

We now turn to (2). Let $\mathcal{Y}=\mathcal{B} \cup \mathcal{C} \cup \mathcal{D} \cup \mathcal{E}$. We wish to show that $\mathcal{Y}$ is closed under coin adjunction.

Suppose first $U \in \mathcal{C}, U=\left(1, a, b, \ldots, c_{k-1}, c_{k}\right)$. Let $W=\left(1, a, b, \ldots, c_{k-1}, c_{k}, c_{k+1}\right)$. We consider two cases, depending upon whether whether $U$ falls under Case A or Case $\mathrm{B}$, above. Suppose $U$ falls under Case A (where $c_{k-1}+a$ is a counterexample for $U$ ). We can assume, by $(*)$, that $c_{k+1} \leq c_{k-1}+a$. It then follows on subtracting $c_{k}$, that $c_{k+1}-c_{k} \leq c_{k-1}-c_{k}+a$. But, if $W \notin \mathcal{C}$, we can assume that $a \leq c_{k+1}-c_{k}$. Hence $a \leq c_{k-1}-c_{k}+a$; an obvious contradiction, since $c_{k}>c_{k-1}$. This shows that any single adjunction to a coin set $U, U \in \mathcal{C}$, under Case A gives rise to a coin set in $\mathcal{C}$ or to a trivial obstruction (in $\mathcal{E}$ ).

Assume now $U$ is an instance of Case $\mathrm{B}$, that is, assume $c_{k}-c_{k-1}=a-1$. We can also assume by $(*)$ that $c_{k+1} \leq c_{k-1}+b$, the counterexample for $U$ in Case $\mathrm{B}$ (otherwise 
$W \in \mathcal{E})$. Hence if $W \notin \mathcal{C}$, then $a \leq c_{k+1}-c_{k} \leq b+c_{k-1}-c_{k}$. But, $b+c_{k-1}-c_{k}=b-a+1$. Hence ( $\dagger) a \leq c_{k+1}-c_{k} \leq b-a+1$. Also, as shown above, $c_{k-1}+b$ is the counterexample for $U$ under Case B. Therefore $W \in \mathcal{D}$. This shows that any coin set in $\mathcal{C}$ under coin adjunction gives rise to a coin set in $\mathcal{Y}$.

Assume now $U=\left(1, a, b, \ldots, c_{k-1}, c_{k}\right)$ and $U \in \mathcal{B}$, that is $c_{k}-c_{k-1}=b-a$. Let $W=\left(1, a, b, \ldots, c_{k-1}, c_{k}, c_{k+1}\right)$. First, as we have shown under $(1), c_{k-1}+(m-1) a$ is a counterexample for $U$. Then by $\left({ }^{*}\right), c_{k+1} \leq c_{k-1}+(m-1) a$. If $W \in \mathcal{C}$, then $W \in \mathcal{Y}$; thus assume that $W \notin \mathcal{C}$; hence, $c_{k+1}-c_{k} \geq a$. Therefore, $a \leq c_{k+1}-c_{k} \leq c_{k-1}+(m-1) a-c_{k}=$ $(m a-b)$, since $c_{k-1}-c_{k}=a-b$. However, $a \leq m a-b$ contradicts the minimality of $m$. Therefore either $W \in \mathcal{C}$ or $W \in \mathcal{E}$ which implies $W \in \mathcal{Y}$.

Finally, assume that $U \in \mathcal{D}$, where $U=\left(1, a, b, \ldots, c_{k}, c_{k+1}\right)$. Thus $(\dagger) a \leq c_{k+1}-$ $c_{k} \leq b-a+1$ and $\left(1, a, b, \ldots, c_{k}\right)$ is non-greedy with counterexample $c_{k-1}+b$. Let $W=\left(1, a, b, \ldots, c_{k}, c_{k+1}, c_{k+2}\right)$. We can assume $W \notin \mathcal{C}$; hence $a \leq c_{k+2}-c_{k+1}$. Moreover, as shown under $(1), c_{k}+b$ is a counterexample for $U=\left(1, a, b, \ldots, c_{k+1}\right)$. Hence, by $\left({ }^{*}\right)$, $c_{k+2} \leq c_{k}+b$. Therefore $a \leq c_{k+2}-c_{k+1} \leq c_{k}+b-c_{k+1}=b+\left(c_{k}-c_{k+1}\right)$. We can further assume that $U \notin \mathcal{C}$, since we have already shown that an adjunction to a coin set in $\mathcal{C}$ leads to a coin set in $\mathcal{Y}$. Hence $a \leq c_{k+1}-c_{k}$. Thus, $a \leq c_{k+2}-c_{k+1} \leq b-a \leq b-a+1$. Thus we have verified $(\dagger)$. Also, since we have already shown under (1) that if $U \in \mathcal{D}$, $U$ is non-greedy with counterexample $c_{k}+b, W \in \mathcal{D}$. This implies $W \in \mathcal{Y}$ and we have completed the proof in all cases.

We next prove two corollaries, the first has already been proven as Theorem 4.1, but we wish to point out that it follows immediately from Theorem 4.5. The second corollary was stated above and although a direct proof can be given, we find it much easier to derive it directly from Theorem 4.5.

Corollary 4.6 Every greedy coin set with four elements is totally greedy.

Proof. Suppose that $(1, a, b, c)$ were greedy but not totally greedy. Since $(1)$ and $(1, a)$ are clearly greedy, it must be that $(1, a, b)$ is not greedy. Then, by Theorem $4.5,(1, a, b)$ is a greedy obstruction which implies that $(1, a, b, c)$ is not greedy!

Corollary 4.7 If $(1, a, b)$ is nongreedy, then so is $(1, a, b, c, d)$ for any integers, $b, c$ with $b<c<d$.

Proof. If $(1, a, b)$ is nongreedy, it is a greedy obstruction; hence no extension of it can be greedy.

\subsection{Some Open Problems}

We have shown that all non-greedy three element coin sets are greedy obstructions, but that there exist four element non-greedy coin sets are not greedy obstructions. It remains an open problem to characterize those non-trivial greedy obstructions with four elements. Same problem for those with $k$ elements, for each integer $k>4$. 
It also would be useful to characterize those coin sets with more than five elements which are greedy but not totally greedy. If an efficient characterization could be obtained, it could lead to a more efficient characterization of greedy coin sets.

Finally, we ask about the asymptotics of the density of the set of totally greedy coin sets within the set of greedy coin sets. If $G(k, R)$ denotes the set of all greedy coin sets

with $k$ coins all of value $\leq R$, and $T G(k, R)$ denotes the set of all totally greedy coin sets with $k$ coins all of value $\leq R$, then what can be said about the ratio $T G(k, R) / G(k, R)$ as $k \rightarrow \infty$ ?

\section{Acknowledgements}

The authors thank Stan Wagon for sharing his computer programs, and thank Jeffrey Shallit for the pointer to Reference [3]. The authors would also like to thank the anonymous referee for numerous suggestions that greatly improved the quality of the presentation of the paper.

\section{References}

[1] D. Knuth. The art of computer programming, volume 2, 3rd edition. Addison Wesley, 1997.

[2] D. Kozen and S. Zaks. Optimal bounds for the change-making problem. Theor. Comput. Sci., 123: 377-388, 1994.

[3] M.J. Magazine, G.L. Nemhauser and L.E. Trotter, Jr. When the greedy solution solves a class of knapsack problems. Operations Research, 23(2): 207-217, 1975.

[4] D. Pearson. A polynomial time algorithm for the change-making problem. Operations Research Letters, 33(3):231-234, 2005.

[5] J. Shallit. What this country needs is an 18-cent piece. Math. Intelligencer, 25(2): 20-23, 2003.

[6] S. Wagon. Greedy coins. http://library.wolfram.com/infocenter/MathSource/5187/

[7] S. Wagon. http://demonstrations.wolfram.com/OptimalityOfGreedyChangeMaking/ 